\title{
Complicaciones de la Mononucleosis infecciosa en la edad adulta. A propósito de un caso clínico.
}

\section{Complications of infectious mononucleosis in adulthood. On the subject of a clinical case.}

\author{
Tirapé Castro Hugo Arturo. ${ }^{1}$, Cajamarca Sacta Lía Cristina. ${ }^{2}$, Villa Cuzco Leonardo \\ Vicente. ${ }^{3} \&$ Uguña Uguña Daniel Esteban ${ }^{4}$
}

\section{Recibido: 10-07-2020 / Revisado: 15-08-2020 /Aceptado: 04-09-2020/ Publicado: 03-10-2020}

DOI: https://doi.org/10.33262/anatomiadigital.v3i4.1472

\begin{abstract}
.
Infectious mononucleosis is a disease caused mainly by the Epstein-Barr virus, it is widespread and it generally occurs in childhood and adolescence, cases in adulthood are rarely reported. Due to its very general characteristics such as thermal rise, pharyngitis and lymphadenopathy, being in most cases self-limited and together with a low incidence of complications in its evolution, it is usually no to diagnosed and it is confused with similar conditions such as the cold. However, there is a small
\end{abstract}

\section{Resumen.}

La mononucleosis infecciosa es una enfermedad ocasionada principalmente por el virus Epstein-Barr, está ampliamente extendida y generalmente se produce en la infancia y la adolescencia, siendo poco común reportar casos en la edad adulta. Debido a sus características muy generales como alza térmica, faringitis y adenopatías, siendo en la mayoría de casos auto limitada y junto a una baja incidencia de complicaciones en su evolución, hacen que sea habitual no diagnosticarla y confundirla con cuadros similares como por ejemplo el

\footnotetext{
${ }^{1}$ Médico Residente Cuidados Intensivos, Hospital Vicente Corral Moscoso, Cuenca, Ecuador, hugo.tirape@gmail.com iD https://orcid.org/0000-0002-5962-3830

${ }^{2}$ Médico en ejercicio libre, Cuenca Ecuador, criss.cajamarca@gmail.es

https://orcid.org/0000-0002-4588-3949

${ }^{3}$ Médico en ejercicio libre, Cuenca Ecuador, (iD https://orcid.org/0000-0001-9727

${ }^{4}$ Médico Residente Emergencias, Hospital Cantonal de Sígsig, Sigsig, Ecuador

(iD) https://orcid.org/0000-0001-5930-949X
} 
percentage of these patients who can have much greater complications and can even lead to death if they are not properly diagnosed and managed, especially when they reach adulthood. Next, a clinical case of atypical mononucleosis is presented, since characteristics such as age, initial manifestations, as well as complications were not the usual ones, which is why it is proposed not to forget the existence of this pathology in differential diagnoses and the importance of an adequate assessment and management.

Keywords: Infectious Mononucleosis, Adult, Complications, Epstein Barr, Hepatic. resfriado común. Sin embargo, existe un pequeño porcentaje de estos pacientes que pueden tener complicaciones mucho mayores e incluso llegar a la muerte si no son adecuadamente diagnosticados y manejados, sobre todo al llegar a la edad adulta. A continuación, se presenta un caso clínico de mononucleosis atípica, ya que características como edad, manifestaciones iniciales, así como las complicaciones no fueron las habituales, motivo por el que se propone no olvidar la existencia de esta patología en los diagnósticos diferenciales y la importancia de una adecuada valoración y manejo.

Palabras claves: Mononucleosis Infecciosa, Adulto, Complicaciones, Epstein Barr, Hepática

\section{Introducción.}

La mononucleosis es una enfermedad infecciosa de origen viral también conocida como la enfermedad del beso debido a que esta es la vía más ampliamente usada por el virus para diseminarse (Méndez-Sánchez, N., Uribe, M., 2004, p. 75), se produce en un 95\% por el virus Epstein-Barr, pudiendo en un porcentaje cercano al 2\% ser producido por Citomegalovirus (Tinoco Racero, I., Caro Gómez, N., Rodríguez Leal, C., López Tinoco, E., 2014, p. 2954) y en un porcentaje aún menor por Toxoplasma gondii (García-Torres, M.L., Primo, J., 2008).

Epidemiológicamente la mononucleosis es una enfermedad ampliamente distribuida a nivel mundial, siendo así que se tiende datos que llevan a pensar que el 95\% de los individuos sanos tienen marcadores serológicos positivos para Epstein-Barr, habiendo cursado la enfermedad sin haberse percatado de la misma (Markin, R.S., 1994, p. 1) (Fica, A., 2003 p. 235) (Angulo, A., Arizcurren, M., Tiberio López, Oteiza Olaso., 2003, p. 311). Esto se da debido a que las características clínicas de la mononucleosis son muy inespecíficas (alza térmica, astenia, odinofagia y presencia de linfadenopatía) lo cual hace fácil la confusión con un proceso gripal común, esto sumado a que la mayor parte de los pacientes tienen una evolución favorable debido a que la enfermedad es autolimitada, dificulta aún más su identificación. (Balfour, H., Dunmire, S., Hogquist., 2015)

Pese a que es una enfermedad bastante común en nuestro entorno y tal vez por la poca incidencia en complicaciones en Ecuador no se tiene un dato estadístico exacto sobre el 
porcentaje de pacientes con marcadores para Epstein-Barr, sin embargo en un estudio realizado por G. Tobar en la ciudad de Quito en el cual de una muestra aleatoria de 85 adolescentes de entre 14 y 15 años determinó que el 96.48\% de los participantes tenían anticuerpos IgG para VEB y apenas un $3.52 \%$ no lo tenían dejando en evidencia que al menos 9 de cada 10 jóvenes se han contagiado antes de cumplir la mayoría de edad. (Balfour et al., 2015)

Se debe considerar que, pese a que las complicaciones no suelen ser frecuentes, estas existen y se encuentran ligadas a diversos factores, siendo uno de los más importantes la edad, ya que mientras más joven sea el paciente menos complicaciones tendrá, esto en relación a aquellos pacientes que se contagian ya en la edad adulta (Littman, A., Rossing, M., Madeline, M., Chen, M., Yasui, Y., 2003, p.728).

\section{Reporte de caso.}

Paciente masculino de 27 años de edad, etnia mestiza, sin hábitos dañinos para la salud de importancia, sin antecedentes patológicos personales relevantes para el caso, madre con hepatopatía no especificada y hermana con artritis reumatoide. Presenta cuadro de 7 días de evolución caracterizado inicialmente por dolor abdominal tipo cólico de moderada intensidad, se acompaña de astenia, hiporexia, alza térmica no cuantificada, odinodisfagia y deposiciones diarreicas en número aproximado de 4 por día en poca cantidad, acude a medico quien diagnostica de gastroenteritis y envía tratamiento a base de Levofloxacina 750mg cada día, mismo que toma por 3 días sin presentar mejoría, 24 horas previo a su ingreso el cuadro de dolor abdominal se exacerba, razón por la cual es valorado en el servicio de emergencias, destaca al examen físico piel con tinte ictérico generalizado, mucosas orales secas, lengua saburral, neurológicamente con tendencia a la somnolencia, en cuello se evidencia presencia de adenopatías cervicales bilaterales de $0.5 \mathrm{~cm}$ de diámetro, no dolorosas no móviles, hemodinámicamente con una frecuencia cardiaca de 130 latidos por minuto, tensión arterial $140 / 100 \mathrm{~mm} / \mathrm{hg}$, saturando sobre $90 \%$ sin soporte de oxígeno complementario, con patrón respiratorio adecuado, el abdomen blando, leve dolor a la palpación profunda en hipocondrio derecho e izquierdo, con ruidos hidroaéreos conservados, diuresis adecuadas, al momento sin alza térmica, se realizan exámenes complementarios evidenciando en ecografía abdominal vesícula distendida de paredes engrosadas, alitiásica, con colédoco permeable, mientras que en la química sanguínea se evidencia aumento de: leucocitos 16200 células $/ \mu 1$, neutrófilos $19.8 \times 10^{3} \mu \mathrm{l}$, linfocitos $74.1 \times 10^{3} \mu \mathrm{l}$, TGO $217.5 \mathrm{U} / \mathrm{L}$, TGP $334.5 \mathrm{U} / \mathrm{L}$, GGT $1425 \mathrm{U} / \mathrm{L}$, bilirrubina directa $6.3 \mathrm{mg} / \mathrm{dl}$, fosfatasa alcalina $206 \mathrm{U} / \mathrm{L}$, se plantean diagnósticos iniciales de colangitis vs síndrome colestásico, equipo quirúrgico decide realizar colangiopancreatografía retrógrada endoscópica, durante el procedimiento encuentran vía biliar de aproximadamente $0.5 \mathrm{~cm}$ permeable, sin imágenes endoluminales, pese a ello se coloca stent, proceden a iniciar terapia antibiótica a base de Piperacilina + Tazobactam 4,5 gramos IV cada 6 horas, sin embargo se evidencia que los valores de transaminasas continúan 
en aumento con bilirrubina directa $(8 \mathrm{mg} / \mathrm{dL})$, se realiza exámenes serológicos para hepatitis con resultados negativos, por lo cual el servicio de cirugía interconsulta a reumatología bajo sospecha de hepatitis autoinmune quien solicita los marcadores correspondientes (ANAS, AMA-M2), panel hepático autoinmune, panel gastrointestinal autoinmune y complementos $\mathrm{C} 3$ y $\mathrm{C} 4$, siendo todos estos resultados negativos.

El deterioro hepático se exacerba, mediado por elevación de bilirrubina directa a $9.6 \mathrm{mg} / \mathrm{dL}$ y se suma al cuadro descenso sostenido de los valores de sodio sérico los cuales no se mantienen normales tras realizar correcciones rápidas, siendo necesario nuevas correcciones diarias, por lo cual es valorado con el servicio de endocrinología que cataloga al paciente como afectado por un SIHAD (secreción inadecuada de la hormona antidiurética) y se indica dejarlo con restricción hídrica, se interconsulta al servicio de cuidados críticos, quienes disponen sea recibido en el servicio de terapia intermedia, se realizan exámenes complementarios como determinación de cortisol, electrolitos en orina, T3 - T4 - TSH, además de tomografía abdominal contrastada en busca de masas tumorales, así como marcadores tumorales en sangre, siendo todos estos negativos.

El paciente va deteriorando su estado y se suma ahora presencia de calambres generalizados continuos, además se evidencia que los valores de TGO y TGP continúan incrementándose en los días posteriores, (TGO 516 U/L - TGP 514 U/L) se consulta al servicio de Gastroenterología quien ante la duda del procedimiento inicial CPRE solicita la realización de una Resonancia Magnética de vías biliares, reportándose normal, el paciente persiste con cuadros febriles sin mejoría desde su ingreso, por lo cual se interconsulta al servicio de infectología quienes tras valoración solicitan prueba de Epstein Barr, siendo este positivo se maneja a paciente con hidratación parenteral, adicionalmente uso de vitamina A por vía oral, con lo cual presenta una evolución favorable en un lapso de 4 días, con posterior disminución de los valores de enzimas hepáticas, después de 12 días de hospitalización, y 2 en el área de cuidados intermedios paciente es dado de alta en mejores condiciones y bajo vigilancia epidemiológica.

\section{Revisión de Literatura.}

Dentro de las posibles complicaciones patológicas de la mononucleosis infecciosa está el compromiso hepático, específicamente la hepatitis, que suele cursarse de forma leve y auto limitada, sobre todo en los pacientes inmunocompetentes, esto es debido a que uno de los principales virus causantes de la mononucleosis infecciosa es el virus de Epstein Barr, el mismo que durante su proliferación tiende también a afectar las células hepáticas. En caso de que la inflamación se vuelva persistente puede deberse al síndrome de infección crónica activa por virus de Epstein Barr. (Cisneros-Herreros, J.M., Herrero-Romero, M., 2006, p. 392). 
La afectación hepática se evidencia por un leve aumento de transaminasas, tanto ALT como AST, el cuadro no cursa de forma agresiva por lo que no amerita de una biopsia hepática, y solo el 5\% desarrollará una hepatitis aguda colestásica. En ocasiones pudiera presentar conjuntamente linfocitos atípicos en sangre periférica así como anticuerpos inmunoglobulina M contra la cápside VCA, esto atribuido a toxicidad ocasionada por la ingesta de antiinflamatorios no esteroideos como por ejemplo el ibuprofeno. (Barreales, M., Pérez Carreras, M., Meizoso, T., Garrido, M., Masedo, A., Colina, F., Solís, J.A., 2006, p.483).

\section{Tratamiento}

Aunque en la actualidad no exista un tratamiento curativo de la mononucleosis infecciosa, podemos incluir cierta terapia que según la bibliografía consultada y estudios realizados aportan un grado válido de mejoría clínica sea en cuanto a síntomas o duración de la enfermedad y carga vírica. La mononucleosis infecciosa no complicada responde de forma adecuada al tratamiento sintomático en la mayoría de los casos, tales medidas son líquidos, dieta blanda y evitar realizar ejercicio por un tiempo aproximado de un mes mientras se resuelve la esplenomegalia (en el caso de que esta sea confirmada) para evitar complicaciones como la ruptura de la misma. (Rezk, E., Nofal, Y.H., Hamzeh, A., Aboujaib, M.F., AlKheder, M.A., Al Hammad, M.F., 2015)

Asociado a estas medidas generales se puede incluir como tratamientos farmacológicos que ayuden al alivio de los síntomas más comúnmente presentados en la enfermedad a medicamentos tales como analgésicos y antipiréticos por ejemplo paracetamol o ibuprofeno enfocados para manejo de alzas térmicas, inflamación y dolor.

Hay que tener especial cuidado con el uso de ácido acetil salicílico ya que al tratarse de una enfermedad vírica es posible el riesgo de síndrome de Reye. (Rezk et al. 2015)

\section{Corticosteroides}

En el estudio "Esteroides para el control de síntomas de mononucleosis infecciosa" publicado en el año 2015 el tratamiento con esteroides alivió el dolor de garganta a corto plazo (a las 12 horas). Los investigadores notaron un beneficio a los dos o cuatro días cuando los esteroides se usaron en combinación con un fármaco antiviral, pero estos hallazgos son limitados ya que se evaluaron en uno o dos ensayos solamente.

Los hallazgos sobre el efecto de los esteroides solos o cuando se usan con un fármaco antiviral para otros síntomas fueron menos claros. Como conclusión se obtuvo que no hay evidencia suficiente de la eficacia de los esteroides para controlar los síntomas de la mononucleosis infecciosa. Hay una falta de investigación sobre los efectos secundarios y las complicaciones a largo plazo. (Rezk et al. 2015) 


\section{Antivirales}

En el estudio "agentes virales para la mononucleosis infecciosa" se incluyeron siete ECA con un total de 333 participantes en la revisión, los antivirales de los estudios incluidos fueron: aciclovir, valomaciclovir y valaciclovir. El seguimiento osciló entre 20 días y seis meses. El diagnóstico de mononucleosis infecciosa se basó en los síntomas clínicos y los parámetros de laboratorio. La calidad de las pruebas se calificó como muy baja para todos los resultados por lo que estos deben interpretarse con cuidado. Se evidencio que hubo una reducción media en la "duración de la linfadenopatía" de nueve días (IC del 95\%: -11,75 a -6,14; dos estudios, 61 participantes) a favor del grupo de tratamiento. En cuanto a la replicación viral, el efecto general de seis estudios fue la supresión de la replicación al administrar tratamiento con antivirales, aunque este efecto no se mantuvo al interrumpir el tratamiento. Para todos los otros resultados, no hubo diferencias estadísticamente significativas entre los grupos de tratamiento antiviral y de control. (De Paor, M., O'Brien, K., Fahey, T., Smith, S.M., 2016).

Como conclusiones los autores manifiestan que la efectividad de los agentes antivirales (aciclovir, valomaciclovir y valaciclovir) en la mononucleosis infecciosa aguda es incierta. La calidad de las pruebas es muy baja. La mayoría de los estudios incluidos estuvo en riesgo de sesgo poco claro o alto por lo cual aún hay interrogantes acerca de la efectividad de esta intervención. (De Paor et al. 2016).

\section{Discusión.}

La mononucleosis infecciosa es una enfermedad ampliamente distribuida a nivel mundial, con mayor incidencia en países en vías de desarrollo y en Ecuador su presencia está dada mayormente en la infancia y la adolescencia presentando anticuerpos $\mathrm{IgG}$ en 8 de cada 10 personas antes de cumplir los 16 años (Méndez-Sánchez, N., Uribe, M., 2004, p. 75). Al ser una enfermedad que en la mayoría de ocasiones se presenta como autolimitada y con una sintomatología muy poco específica es común que pase desapercibida. (Barreales, M., Pérez -Carreras, M., Meizoso, T., Garrido, M., Masedo, A., Colina, F., Solís, J.A., 2006, p. 483) En el caso presentado se evidencia por tanto una de esas excepciones a la regla, pues el paciente era un adulto, quien debuta con una sintomatología distinta a la habitual y presenta varias complicaciones que luego en retrospectiva parecieran ser bastante obvias, sin embargo a su debido momento causaron confusión al personal de salud, siendo este caso un ejemplo de como en ocasiones una enfermedad con un manejo inadecuado puede terminar complicando e incluso comprometiendo la integridad de un paciente.

El paciente ingresa con un cuadro de 7 días de dolor abdominal, alza térmica y deposiciones diarreicas, lo cual de entrada no nos sugiere por los síntomas como parte de los diagnósticos diferenciales a la mononucleosis infecciosa y al momento de su ingreso el paciente suma al cuadro tinte ictérico lo cual sugiere al personal de salud la patología colangitis (triada de Charcot) (Camacho, J.F., 2012, p. 148), se realizan los exámenes complementarios y 
encuentran en ellos elevación de las transaminasas, bilirrubinas elevadas y formula leucocitaria alta lo cual casi confirma la sospecha de los especialistas de una colangitis esto sumado a una ecografía que muestra una vesícula distendida de paredes gruesas, lo que lleva al personal médico a decidir realizar una CPRE, para sorpresa del equipo médico la vesícula está libre y es de paredes conservadas, esto debido en gran manera a que la patología vesicular aguda es una entidad muy común dentro de los diagnósticos diferenciales de dolor abdominal, y si bien la ultrasonografía es uno de los métodos más fácilmente accesibles no podemos olvidar que este es un método operador-dependiente lo cual aumenta la probabilidad de confusión. (Motta Ramírez, G., Rodríguez Treviño, C., 2010).

Los valores de transaminasas así como bilirrubinas siguieron aumentando, otra sospecha diagnostica que se barajo fue la posibilidad de una Hepatitis Toxica producida por los medicamentos utilizados previo ingreso del paciente, ya que esta patología suele presentarse tras la administración de fármacos y exacerbar al poco tiempo sin dar cambios estructurales a nivel hepático (Navarro, V., Senior, J., 2006, p. 354), sin embargo al realizar revisar en los antecedentes del paciente este ya había tomado dicha medicación previamente sin haber presentado ningún tipo de alteración (Andrade, R.J., Tulkens, P.M., 2011, p.1431), de igual manera se descartó algún tipo de hepatitis alcohólica ya que el paciente era abstemio y jamás había consumido alcohol en su vida, razón por lo cual el equipo médico decide realizar exámenes complementarios bajo sospecha de una hepatitis autoinmune y pese a que este es más frecuente en mujeres (García-Torres, M.L., Primo, J., 2008, p.400), no se puede descartar la posibilidad de su presencia ante un caso en el cual se han desechado ya otras patologías más comunes, sin embargo estas también fueron negativas.

A este caso se suma hiponatremia, los valores de sodio no se recuperan pese a las correcciones que se realizan, esto confunde aún más al personal de salud ya que siempre se orienta primero a pensar en una misma enfermedad que produce dos síntomas antes de pensar en dos enfermedades distintas en el mismo paciente, sin embargo bajo sospecha de SIHAD como enfermedad de descarte (Tensiones arteriales normales, Densidad urinaria dentro de rangos y aumento del sodio urinario), endocrinología sugiere restricción hídrica como tratamiento de base, lo cual desencadena un aumento en las enzimas hepáticas y en una descompensación del paciente acompañada por calambres continuos y dolor generalizado. (Runkle \& Villabona, 2014, p.425)

Se reinstaura proceso febril y con un paciente ya en cuidados intermedios se realizan pancultivos siendo todos estos negativos, una valoración integral del paciente y una vista en retrospectiva nos permite revalorar al mismo y buscar nuevas opciones diagnósticas. Considerando la fiebre, los ganglios inflamados, la odinofagia y el posterior deterioro hepático del paciente, en conjunto con el servicio de infectología se decide realizar examen de Epstein Bar, dando este un resultado positivo, se reinicia hidratación amplia a paciente y los valores hepáticos mejoran considerablemente. (Méndez-Sánchez \& Uribe, 2004, p.75) 


\section{Conclusiones:}

En el caso presentado de un paciente adulto sin hábitos ni antecedentes de importancia se suscita un cuadro hepático que lo lleva a permanecer en el ámbito hospitalario durante 12 días, recibiendo múltiples exámenes e incluso procedimientos invasivos, para posteriormente ser diagnosticado de Mononucleosis Infecciosa, llevándonos a las siguientes conclusiones:

- Nos recuerda que la medicina no siempre es solo la suma síntomas y signos, sino el proceso lógico y estratificado de encontrar la causa del problema sin olvidar la atipicidad de cada patología.

- La importancia fundamental de la historia clínica detallada, exhaustiva no solo en los síntomas sino también en los factores de riesgo dentro de los tiempos acordes a las patologías probables.

- El trabajo del equipo médico multidisciplinario en los pacientes con cuadros atípicos es fundamental para considerar los diferentes enfoques y caminos a seguir en pos de descubrir y lograr la curación de las diferentes patologías de difícil resolución.

\section{Referencias Bibliographical.}

Andrade, R.J., Tulkens, P.M. (2011) Hepatic safety of antibiotics used in primary care. J Antimicrob Chemother, 66(7), 1431-1446 https://doi.org/10.1093/jac/dkr159

Angulo, A., Arizcurren, M., Tiberio López, Oteiza Olaso. (2003) Clinical and analytic characteristics and complications in patients with infectious mononucleosis referred from primary care to specialists. Atención Primaria, 311.

Balfour, H., Dunmire, S., Hogquist. (2015) Infectious mononucleosis: clin transl immunology. http://www.nature.com/cti/journal/v4/n2/pdf/cti20151a.pdf.

Barreales, M., Pérez -Carreras, M., Meizoso, T., Garrido, M., Masedo, A., Colina, F., Solís, J.A. (2006) Infección por el virus de Epstein-Barr y hepatitis aguda colestásica. Servicios de Medicina Aparato Digestivo y Anatomía Patológica. Hospital Universitario. AN. MED. INTERNA (Madrid), Vol. 23, N. ${ }^{\circ}$ 10, 483-486.

Camacho, J.F. (2012) Historia y filosofía de la medicina: Charcot y su legado a la medicina. Gaceta Médica de México, 6, 148-321.

Cisneros-Herreros, J.M., Herrero-Romero, M. (2006) Hepatitis por virus del grupo herpes. Enferm Infecc Microbiol Clin, 24, 392-398.

7De Paor, M., O'Brien, K., Fahey, T., Smith, S.M. (2016) Antiviral agents for infectious mononucleosis (glandular fever). Cochrane Database of Systematic Reviews, 12, CD011487 https://doi.org/10.1002/14651858.CD011487 
Fica, A. (2003) Síndrome de mononucleosis infecciosa en pacientes adolescentes y adultos. Revista Chilena de Infectología, 20, 235-242.

García-Torres, M.L., Primo, J. (2008) Estudio clínico de la hepatitis autoinmune del adulto en Valencia, REV ESP ENFERM DIG (Madrid) Vol. 100, 400-404.

Littman, A., Rossing, M., Madeline, M., Chen, M., Yasui, Y. (2003) Association between late age at infectious mononucleosis, Epstei-Barr virus antibodies, and ovarian cancer risk. Scandinavian Journal of Infectious Disease, Vol. 35, 728-735.

Markin, R.S. (1994) Manifestations of Epstein-Barr virus-associated disorders in liver. Liver, 14, 1-13.

Méndez-Sánchez, N., Uribe, M., (2004) Infectious mononucleosis hepatitis: a case-report. Ann Hepatol, 3, 75-76.

Motta Ramírez, G., Rodríguez Treviño, C. (2010) Abordaje diagnóstico por imagen en patología benigna de la vesícula y vías biliares. CIRUGÍA ENDOSCÓPICA, Vol.11, No. 2.

Navarro, V., Senior, J. (2006) Drug-related hepatotoxicity. New England Journal of Medicine, 9, 354-731.

Rezk, E., Nofal, Y.H., Hamzeh, A., Aboujaib, M.F., AlKheder, M.A., Al Hammad, M.F. (2015) Steroids for symptom control in infectious mononucleosis. Cochrane Database of Systematic Reviews, 11, CD004402 http://doi.org/10.1002/14651858.CD004402

Rubalcava Lara, L.F., Tello Casillas, J.K. (2019) Mononucleosis Epstein-Barr negativa, variación poco reconocida de enfermedad popular. Revista de la Facultad de $\begin{array}{llllll}\text { Medicina de la } & \text { UNAM, }\end{array}$ http://doi.org/10.22201.fm.24484865e.2019.62.2.06

Runkle, S., Villabona, C. (2014) Tratamiento de la hiponatremia secundaria al síndrome de secreción inadecuada de la hormona antidiurética: algoritmo multidisciplinar español. Sociedad española de nefrología, Vol. 34, Núm. 4, 425-544.

Tinoco Racero, I., Caro Gómez, N., Rodríguez Leal, C., López Tinoco, E. (2014) Infecciones por el virus de Epstein-Barr y citomegalovirus. Rev. Medicine Madrid, 11, 29542964.

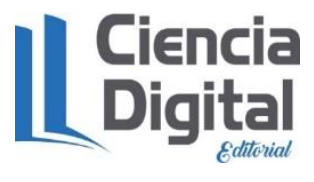




\section{PARA CITAR EL ARTÍCULO INDEXADO.}

Tirapé Castro , H. A., Cajamarca Sacta , L. C., Villa Cuzco , L. V., \& Uguña Uguña , D. E. (2020). Complicaciones de la Mononucleosis infecciosa en la edad adulta. A propósito de un caso clínico. Anatomía Digital, 3(4), 68-77. https://doi.org/10.33262/anatomiadigital.v3i4.1472

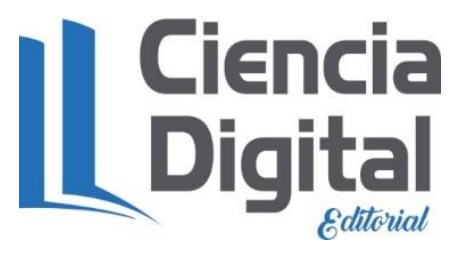

El artículo que se publica es de exclusiva responsabilidad de los autores y no necesariamente reflejan el pensamiento de la Revista Anatomía Digital.

El artículo queda en propiedad de la revista y, por tanto, su publicación parcial y/o total en otro medio tiene que ser autorizado por el director de la Revista Anatomía Digital.
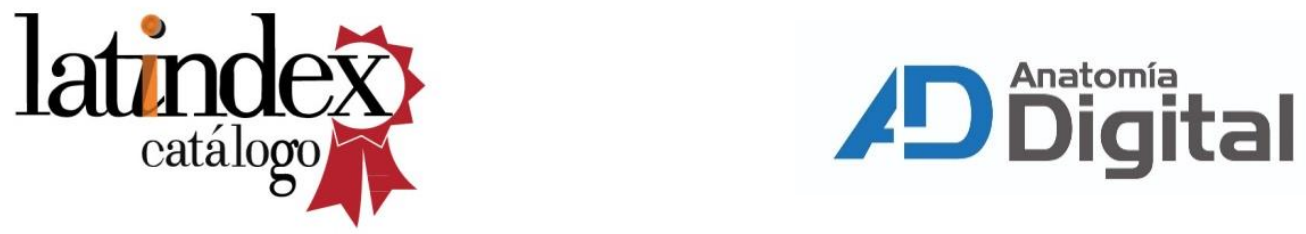